\title{
An electronic structure descriptor for oxygen reactivity at metal and metal-oxide surfaces
}

Dickens, Colin F.; Montoya, Joseph H.; Kulkarni, Ambarish R.; Bajdich, Michal; Nørskov, Jens K.

Published in:

Surface Science

Link to article, DOI:

10.1016/j.susc.2018.11.019

Publication date:

2019

Document Version

Peer reviewed version

Link back to DTU Orbit

Citation (APA):

Dickens, C. F., Montoya, J. H., Kulkarni, A. R., Bajdich, M., \& Nørskov, J. K. (2019). An electronic structure descriptor for oxygen reactivity at metal and metal-oxide surfaces. Surface Science, 681, 122-129. https://doi.org/10.1016/j.susc.2018.11.019

\section{General rights}

Copyright and moral rights for the publications made accessible in the public portal are retained by the authors and/or other copyright owners and it is a condition of accessing publications that users recognise and abide by the legal requirements associated with these rights.

- Users may download and print one copy of any publication from the public portal for the purpose of private study or research.

- You may not further distribute the material or use it for any profit-making activity or commercial gain

- You may freely distribute the URL identifying the publication in the public portal 


\title{
An electronic structure descriptor for oxygen reactivity at metal and metal- oxide surfaces
}

\author{
Colin F. Dickens ${ }^{\mathrm{a}, \mathrm{b}}$, Joseph H. Montoya ${ }^{\mathrm{c}}$, Ambarish R. Kulkarni ${ }^{\mathrm{a}, \mathrm{d}}$, Michal Bajdich ${ }^{\mathrm{b}}$, \\ Jens K. Nørskov ${ }^{\mathrm{a}, \mathrm{b}, \mathrm{e}, *}$ \\ ${ }^{a}$ SUNCAT Center for Interface Science and Catalysis, Department of Chemical Engineering, Stanford University, Shriram Center, 443 Via Ortega, Stanford, CA 94305 , \\ $U S A$ \\ ${ }^{\mathrm{b}}$ SUNCAT Center for Interface Science and Catalysis, SLAC National Accelerator Laboratory, 2575 Sand Hill Road, Menlo Park, CA 94025, USA \\ cEnergy Technologies Area, Lawrence Berkeley National Laboratory, Berkeley, CA 94720, USA \\ ${ }^{\mathrm{d}}$ Department of Chemical Engineering, University of California, Davis, CA 95616, USA \\ ${ }^{\mathrm{e}}$ Department of Physics, Technical University of Denmark, 2800 Kongens Lyngby, Denmark
}

\begin{abstract}
A B S T R A C T
Identifying and understanding relationships between the electronic and atomic structure of surfaces and their catalytic activity is an essential step towards the rational design of heterogeneous catalysts for both thermal and electrochemical applications. Herein, we identify a relationship between the atom-projected density of states of surface oxygen and its ability to make and break bonds with the surrounding metal atoms and hydrogen. This structure-property relationship is shown to hold across different classes of materials (metals, rutile metal-oxides, and perovskite metal-oxides) and for different oxygen binding sites (i.e. different oxygen coordination numbers). We utilize understanding from the $d$-band model and the simple two-level quantum coupling problem to shed light on the physical origin of this relationship for transition metal surfaces and we hypothesize similar principles extend to the other materials considered. Finally, we demonstrate the utility of the identified descriptor to serve as a tool for high throughput screening of oxygen active sites for large systems where many unique oxygen sites exist and can be computationally expensive to probe individually. As an example, we predict the reactivity of 36 unique oxygen atoms at a kinked $\mathrm{RuO}_{2}$ extended surface from a single self-consistent DFT calculation.
\end{abstract}

\section{Introduction}

The field of heterogeneous catalysis has benefited greatly from physical models relating the catalytic properties of surfaces with their electronic structure. A particularly successful example is the $d$-band model for transition metals, [1-3] which has provided a simple means for rationalizing trends in surface reactivity upon changes in composition (alloying), lattice constant (strain), and facet. [4-7] The $d$-band model has also provided a physical basis for the widely used descriptorbased analyses and scaling relationships between adsorbate binding energies and transition state energies, which are essential tools for catalyst design. [8-10] Building upon understanding provided by the $d$ band model, the generalized coordination number has been shown to be a good atomic structure-based descriptor of site reactivity for pure metals, [11-13] and recently, it has been demonstrated that the binding energy of a surface metal atom itself serves as a robust and continuous descriptor of its reactivity. [14] By including some information about the catalyst's electronic structure (Bader charges, electronegativities, electron hopping integrals), researchers have devised modified coordination number descriptors that can serve as prediction tools for systems beyond pure transition metals including core-shell nanoparticles, single-site catalysts, and transition metal-oxides. [15-19]

In the case of transition metal-oxides, recent efforts have attempted to elucidate a direct relationship linking electronic structure and catalytic properties. Beyond electron-counting based approaches, $[20,21]$ correlations between computed adsorbate binding energies and either the metal $d$-states or oxygen $2 p$-states have been made with some success for rutile, perovskite, and rock-salt transition metal-oxides. [22-27] The experimental oxygen electrochemistry rates of perovskite catalysts, which are a function of their adsorbate binding energies, have also been shown to correlate with formal electron occupancies and other electronic structural properties such as the oxygen $2 p$-band center and charge-transfer energy that were extracted from density functional theory (DFT) calculations or X-ray spectroscopy measurements.

\footnotetext{
* Corresponding author at: Department of Physics, Technical University of Denmark, 2800 Kongens Lyngby, Denmark

E-mail address: jkno@dtu.dk (J.K. Nørskov).
} 
[28-32] Insight into the covalency between metal and oxygen has also been obtained for lithium cobalt oxide by probing oxygen excited states by electron and X-ray spectroscopy measurements. [33,34] Despite these observed correlations, the field has yet to develop a simple, physical framework based on chemical bonding theory for relating the electronic structure and binding properties of oxide catalysts akin to the $d$-band model for transition-metals.

In this work, we add to the discussion by examining the reactivity and electronic structure of adsorbed oxygen, rather than the metal atom to which it binds. The reactivity of surface oxygen is an important descriptor for oxidation chemistries such as the oxygen evolution reaction (OER) and methane activation. [35-37] We present a correlation between the reactivity and projected density of states (PDOS) of surface oxygen from DFT calculations that holds across metals and metal-oxides and for different oxygen binding sites (i.e. different oxygen coordination numbers). We provide a physical interpretation of the correlation in the case of transition-metals, and we also demonstrate the utility of the electronic structural descriptor in screening for active sites. Specifically, we will examine the case of a kinked $\mathrm{RuO}_{2}$ surface, which is relevant for the OER.

\section{Theoretical methods}

This work consists of DFT calculations of FCC transition metal, rutile metal-oxide, and perovskite metal oxide surfaces. Perovskite structures were obtained from ref. [27] and in some cases were re-optimized as described in the Supplementary Information. The exact computational details vary for each of these data sets and a detailed explanation of each is provided in the Supplementary Information. In general, a bulk optimization was performed for each material before cutting the surface. Surface geometries consisting of adsorbed $\mathrm{O}$ and $\mathrm{OH}$ were optimized and the lower surface layers were fixed to their bulk positions. All calculations were performed using the planewave code, Quantum Espresso via the Atomic Simulation Environment python interface. $[38,39]$ Exchange and correlation were treated with either the RPBE or BEEF-vdW functionals, $[40,41]$ and spin-polarization was neglected. We note that the inclusion of higher-level methods (GGA + U or hybrid functionals) and spin-polarization is necessary to achieve an accurate physical description of a subset of the materials considered in this work. For this reason, we avoid making claims about the behavior of any single material, and instead focus on general trends and correlations.

\section{Results and discussion}

\subsection{Correlation between the average 2p-state energy and reactivity of adsorbed oxygen}

The primary catalytic property of interest in this study is the energy required to deprotonate adsorbed $\mathrm{OH}$ to create adsorbed $\mathrm{O}$

$\Delta E_{\mathrm{O}}-\Delta E_{\mathrm{OH}}=E_{\mathrm{O} *}+\frac{1}{2} E_{\mathrm{H}_{2}(\mathrm{~g})}-E_{\mathrm{OH} *}$.

This quantity is simply the negative of hydrogen binding energy at an oxygen site and is thus indicative of the oxygen atom's reactivity. We have elected to use the term $\Delta E_{\mathrm{O}}-\Delta E_{\mathrm{OH}}$ rather than $-\Delta E_{\mathrm{H}}$ to be consistent with the OER community where $\Delta G_{\mathrm{O}}-\Delta G_{\mathrm{OH}}$ is the conventional activity descriptor. Additionally, this terminology explicitly indicates that hydrogen is adsorbing/desorbing to/from an oxygen atom rather than a metal atom.

The electronic structure-based descriptor used in this study will be referred to as the average $\mathrm{O} 2 p$-state energy and is calculated as the first moment of the DOS projected onto the atomic $2 p$-states of an adsorbed oxygen atom (after deprotonation) relative to the Fermi level $\bar{\varepsilon}_{2 \mathrm{p}}=\frac{\int_{\varepsilon_{\min }}^{\varepsilon_{\max }} \rho_{2 \mathrm{p}} \varepsilon d \varepsilon}{\int_{\varepsilon_{\min }}^{\varepsilon_{\max }} \rho_{2 \mathrm{p}} d \varepsilon}$

For the systems examined in this work, we generally find the $\mathrm{O} 2 p$ states to lie above $-10 \mathrm{eV}$ relative to the Fermi level, so this value is chosen as our lower energy bound, $\varepsilon_{\min }$. The choice of an upper energy bound, $\varepsilon_{\max }$, is more ambiguous because it is unclear a priori how many of the unoccupied states are chemically relevant for the transformation between adsorbed $\mathrm{O}$ and adsorbed $\mathrm{OH}$. Additionally, the quantity of unoccupied states present depends on the system and the number of bands used in the DFT calculation. For the systems considered in this work, we find that choosing $\varepsilon_{\max }$ values between 1 and $2 \mathrm{eV}$ relative to the Fermi level minimizes the model error as shown in Fig. S1, suggesting that unoccupied states within this range of the Fermi level play an important chemical role. Ultimately, all reported results use an $\varepsilon_{\max }$ of $2 \mathrm{eV}$ relative to the Fermi level. We purposefully avoid use of the term "O $2 p$-band center" because in many cases the distribution of $\mathrm{O} 2 p$ states consists of discrete bonding and antibonding states rather than a continuous band of states as will be shown later.

Fig. 1a shows a correlation between the average $O 2 p$-state energy, $\bar{\varepsilon}_{2 p}$, and $\Delta E_{\mathrm{O}}-\Delta E_{\mathrm{OH}}$ for oxygen atoms at various metal and metal-oxide surfaces. The data in Fig. 1a consists of face-centered-cubic (FCC) metal (111) surfaces, rutile metal-oxide (110) surfaces, and cubic perovskite (100) surfaces (from Ref. [27]) and is broken down by each material class in Fig. 1b-d. For each point in Fig. 1, $\bar{\varepsilon}_{2 p}$ (Eq. (2)) of a specific oxygen atom is compared to the independently calculated $\Delta E_{\mathrm{O}}-\Delta E_{\mathrm{OH}}$ (Eq. (1)) for the same oxygen atom. This means that there may be multiple data points for a single surface, one for each unique oxygen binding site as illustrated in Fig. 1b-d. The coloring of the points is related to the group of the metal that $\mathrm{O}$ is bound to as indicated by the inset periodic table. We acknowledge that not all surfaces in Fig. 1 are practically relevant, but the range of compositions and structures serves to demonstrate the robustness of the correlation. More details regarding the DFT calculations can be found in the Supplementary Information.

Fig. 1 shows that bound oxygen atoms with higher-lying $2 p$-states have higher $\Delta E_{\mathrm{O}}-\Delta E_{\mathrm{OH}}$, or equivalently, stronger affinities towards binding hydrogen. In general, oxygen atoms with higher coordination to metal atoms tend to have weaker affinities towards binding hydrogen. The dataset as a whole has a near-unity slope, meaning that variations in $\bar{\varepsilon}_{2 p}$ translate directly into variations in $\Delta E_{\mathrm{O}}-\Delta E_{\mathrm{OH}}$. This is also true for either metal-oxide dataset on their own, but the FCC metals are best fit with a lower slope of 0.79 as shown in Fig. 1b-d. The five particularly bad outliers (circled in Fig. 1a) are oxygen atoms in the $\mathrm{MO}_{2}$ plane at $\mathrm{Hf}$ and $\mathrm{Zr}$-based perovskite surfaces. These data points are somewhat unique in that they have no density of states at the Fermi level in the post-deprotonation (oxygen adsorbed) state. The only other such data points are for two and three-fold coordinated oxygen at the rutile $\mathrm{TiO}_{2}$ surface, both of which fall within the scatter of the other data.

$\mathrm{Xu}$ and Kitchin have published a similar correlation between the average $\mathrm{O} 2 p$-state energy, $\bar{\varepsilon}_{2 p}$, and oxygen chemisorption energy, $\Delta E_{\mathrm{O}}$, for six $3 d$ transition-metals and their rock-salt oxide counterparts. [24] We have considered the correlations with $\Delta E_{\mathrm{O}}$ and $\Delta E_{\mathrm{OH}}$ in Figs. S2 and $S 3$, respectively and find that they are significantly weaker than the one with $\Delta E_{\mathrm{O}}-\Delta E_{\mathrm{OH}}$, which is reflective of the imperfect scaling between $\Delta E_{\mathrm{O}}$ and $\Delta E_{\mathrm{OH}}$. Physical rationale for this observation will be provided in Section 3.4.

With regards to OER catalyst screening, the optimal $\Delta G_{\mathrm{O}}-\Delta G_{\mathrm{OH}}$ is in the range of 1.5-1.7 eV based on the conventional mechanism and scaling relations. [35,37] Assuming a constant free energy correction of $-0.3 \mathrm{eV}$ for $\Delta E_{\mathrm{O}}-\Delta E_{\mathrm{OH}}$ (see Supplementary Information for details), the correlation in Fig. 1 suggests that OER-active oxygen atoms have an $\bar{\varepsilon}_{2 p}$ between -2 and $-3 \mathrm{eV}$. 

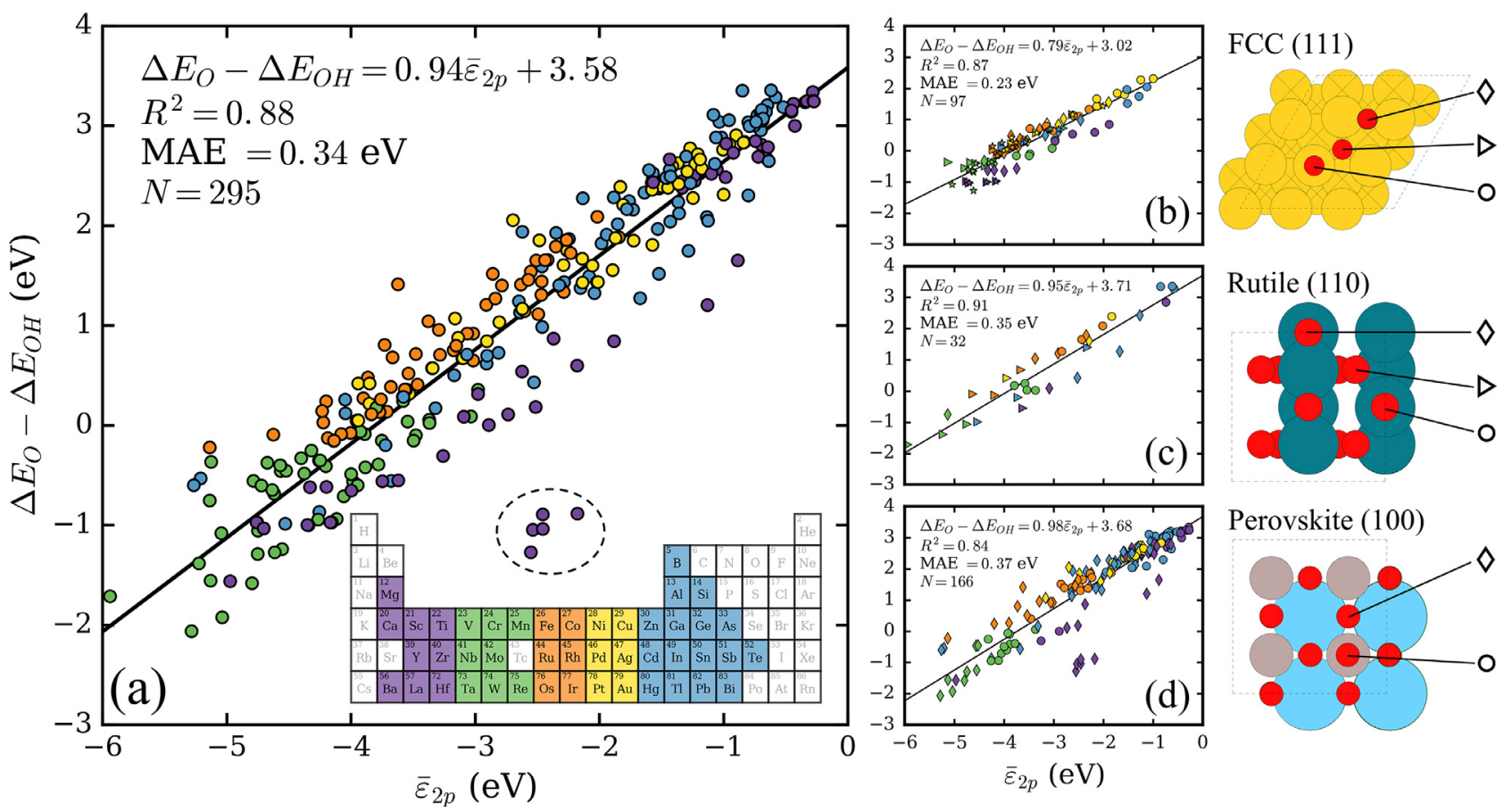

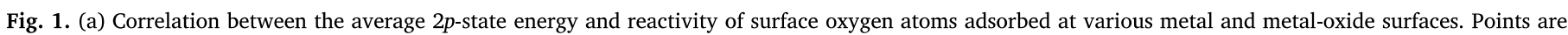
colored based on the identity of the metal atom that oxygen is bound to (see inset periodic table). The five circled outliers are in-plane oxygen at Hf and Zr based perovskite surfaces, which have no density of states at the Fermi level. At right, the data is broken down by material: FCC metal (111) in (b), rutile metal-oxide (110) in (c), and perovskite metal-oxide (100) in (d) with distinct oxygen binding sites represented by different marker shapes as illustrated in the top-down views for each type of surface. In the case of FCC (111) surfaces, star symbols indicate that adsorbed O and OH were allowed to fully relax at their most stable respective sites. In the case of in-plane oxygen atoms at perovskite surfaces (diamonds), coloring is based on the $B$-site cation, which shares a shorter bond with the oxygen. Structures are available as part of the Supplementary Information and via Catalysis-Hub.org. [42]. (For interpretation of the references to color in this figure legend, the reader is referred to the web version of this article.)

\subsection{Evaluating a direct analogy to the d-band model}

Next, we would like to gain some physical rationale for why such a robust correlation exists and why it holds for variations in both catalyst composition and oxygen binding site. At first glance, a direct $d$-band model analogy seems promising. According to the $d$-band model, surfaces with higher-lying $d$-bands relative to the Fermi level (fewer $d$ electrons) are more reactive towards adsorbates. [1,2] This is not unlike the correlation observed in Fig. 1, where oxygen atoms with higher average $2 p$-state energies are more reactive towards binding a hydrogen atom. In the case of the $d$-band model, this trend arises because higherlying $d$-bands lead to higher-lying, and thus less filled, antibonding states, resulting in a stronger metal-adsorbate bond.

To investigate if a similar explanation can be applied to the correlation between the average $2 p$-state energy and reactivity of surface oxygen atoms, we have examined the PDOS of adsorbed $\mathrm{O}$ and $\mathrm{OH}$. Shown in Fig. 2 are the $2 p$-states projected onto adsorbed $\mathrm{O}$ and the $1 s$ states projected onto $\mathrm{H}$ in adsorbed $\mathrm{OH}$ at $\mathrm{Ag}(111)$ and $\mathrm{LaOsO}_{3}(100)$ surfaces, which are relatively weak and strong binding, respectively. For each surface, the bonding states and antibonding states for the $\mathrm{M}-\mathrm{O}$ (double) bond are reflected in the $2 p$-states projected onto adsorbed $\mathrm{O}$. Notice that the $\mathrm{M}-\mathrm{O}$ bonding states are well below the Fermi level (fully occupied), while the $\mathrm{M}-\mathrm{O}$ antibonding states straddle the Fermi level (partially occupied).

Similarly, we can see the bonding and antibonding states for the $\mathrm{O}-\mathrm{H}$ bond reflected in the 1s-states projected onto $\mathrm{H}$ in adsorbed $\mathrm{OH}$. In both cases in Fig. 2, the bonding states of the $\mathrm{O}-\mathrm{H}$ bond are fully occupied (as for the $\mathrm{M}-\mathrm{O}$ bond), but the $\mathrm{O}-\mathrm{H}$ antibonding states are completely unoccupied, resulting in an optimal $\mathrm{O}-\mathrm{H}$ bond strength. Because we see little differences in filling of $\mathrm{O}-\mathrm{H}$ antibonding states between the materials in this study, we hypothesize that the $\mathrm{O}-\mathrm{H}$ bond strength is relatively constant. Further evidence is shown in Fig. S4, which illustrates the narrow range of observed $\mathrm{O}-\mathrm{H}$ bond lengths (0.96-1.02 $\AA$ ). Because $\Delta E_{\mathrm{O}}-\Delta E_{\mathrm{OH}}$ can be considered to be the sum of $\mathrm{O}-\mathrm{H}$ bond cleavage and the formation of a second $\mathrm{M}-\mathrm{O}$ bond, it must therefore be the latter that distinguishes the various surfaces and active sites in Fig. 1. In other words, we interpret Fig. 1 to mean that $\bar{\varepsilon}_{2 p}$ is correlated in some way with the energy of the second $\mathrm{M}-\mathrm{O}$ bond rather than the energy of the $\mathrm{O}-\mathrm{H}$ bond, and we conclude that a direct $d$-band model analogy is not applicable to explain the reactivity of a surface oxygen towards hydrogen as a function of its average $2 p$-state energy.

As noted above and as one might expect based on the observed scaling relationship between $\mathrm{O}$ and $\mathrm{OH}$ binding energies, $[27,43,44]$ there is also some correlation between $\bar{\varepsilon}_{2 p}$ and $\mathrm{O}$ and $\mathrm{OH}$ chemisorption energies, as shown in Figs. S2 and S3, respectively. However, it is important to note that this correlation significantly weaker than the correlation with $\Delta E_{\mathrm{O}}-\Delta E_{\mathrm{OH}}$. By definition, $\Delta E_{\mathrm{O}}$ itself is a function of the $\mathrm{M}-\mathrm{O}$ bond strength, so in addition to explaining the correlation between $\bar{\varepsilon}_{2 p}$ and $\Delta E_{\mathrm{O}}-\Delta E_{\mathrm{OH}}$, the goal of the following discussion is also to elucidate why it is stronger than the correlation between $\bar{\varepsilon}_{2 p}$ and $\Delta E_{\mathrm{O}}$.

\subsection{Relating variations in $\bar{\varepsilon}_{2 p}$ to variations in the $M-O$ bond strength}

We restrict our discussion to transition metals for now, where the understanding yielded by the $d$-band model is at our disposal. Variations in chemical bond strength can be broken up into an attractive hybridization term and a repulsive orthogonalization term (i.e. Pauli repulsion):

$\delta E_{\mathrm{M}-\mathrm{O}} \sim \delta E_{\mathrm{M}-\mathrm{O}}^{\mathrm{hyb}}+\delta E_{\mathrm{M}-\mathrm{O}}^{\mathrm{orth}}$.

As demonstrated previously, [2] variations in surface-adsorbate bond strengths may be correlated with variations in the sum of one electron energies within the frozen potential approximation. In this case, the hybridization term for coupling between the metal $d$-states 


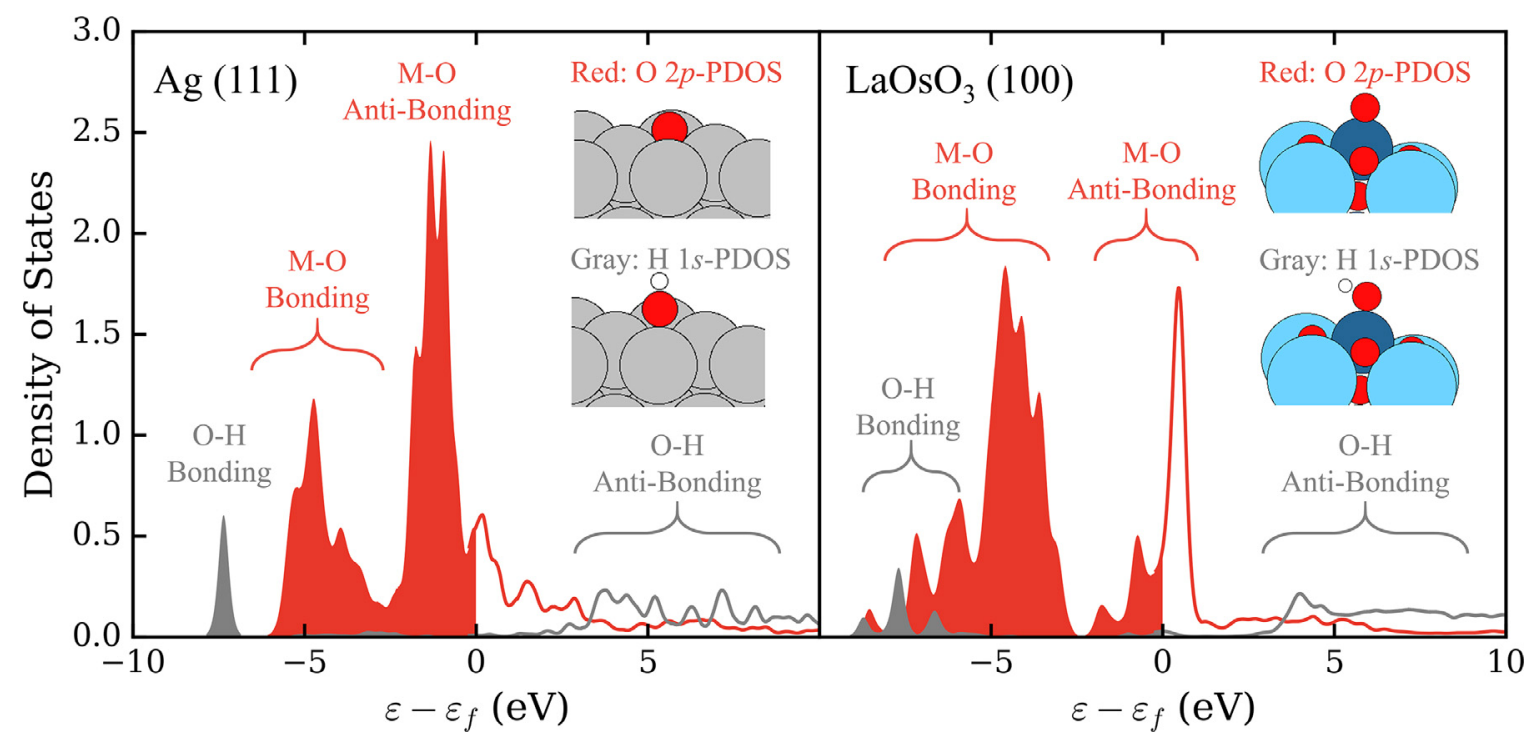

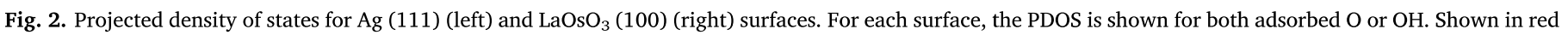

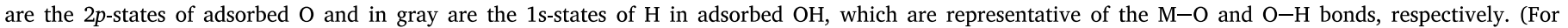
interpretation of the references to color in this figure legend, the reader is referred to the web version of this article.)

and $\mathrm{O} 2 p$-states can be written as

$\delta E_{\mathrm{M}-\mathrm{O}}^{\text {hyb }}=-(1-f) \frac{V_{2 p-d}^{2}}{\varepsilon_{d}-\varepsilon_{2 p}}$

where $f$ (ranging from 0 to 1 ) is the filling of $\mathrm{M}-\mathrm{O}$ antibonding states, $V_{2 p-d}$ is the coupling matrix element for metal $d$-states and oxygen $2 p$ states, $\varepsilon_{d}$ is the center of the $d$-band, and $\varepsilon_{2 p}$ is the center of the renormalized $O 2 p$-states after coupling to the metal's $s p$-band (but before coupling to the metal's $d$-band). This expression is analogous to the simple two-level quantum coupling problem and exploits that the M-O bonding states are always filled. The orthogonalization term can be written as a product of overlap $\left(S_{2 p-d}\right)$ and coupling $\left(V_{2 p-d}\right)$ matrix elements, which are assumed to be proportional to each other:

$\delta E_{\mathrm{M}-\mathrm{O}}^{\mathrm{orth}} \sim\left|S_{2 p-d} V_{2 p-d}\right| \sim V_{2 p-d}^{2}$.

From Eqs. (4) and (5), we reason that the $\mathrm{M}-\mathrm{O}$ bond strength is primarily a function of the $\mathrm{M}-\mathrm{O}$ coupling strength, $V_{2 p-d}$, and the positions of the metal $d$-states and renormalized oxygen $2 p$-states, $\varepsilon_{d}$ and $\varepsilon_{2 p}$, respectively, which together determine $f$, the filling of $\mathrm{M}-\mathrm{O}$ antibonding states. Furthermore, $\varepsilon_{2 p}$ is a function of the $2 p$ state energy of atomic oxygen, which can be assumed to be a constant relative to the Fermi level neglecting differences in work function, and the coupling strength between the $2 p$ states of atomic oxygen and the $s p$ band of the metal, $V_{2 p-s p}$.

In summary, $\delta E_{\mathrm{M}-\mathrm{O}}$ is a function of $\varepsilon_{d}, V_{2 p-d}, V_{2 p \text {-sp. }}$. The two coupling matrix elements are not completely independent of one another, so we broadly conclude that variations in the $\mathrm{M}-\mathrm{O}$ bond strength are a function of variations in the $d$-band center (fingerprint of the metal surface) and in the $\mathrm{M}-\mathrm{O}$ coupling strength (fingerprint of the binding site). Next, we explain why variations in these properties have a similar effect on both $\bar{\varepsilon}_{2 p}$ and $\Delta E_{\mathrm{O}}-\Delta E_{\mathrm{OH}}$.

To probe directly how surface electronic structure and $\mathrm{M}-\mathrm{O}$ coupling strength are reflected in both the $\mathrm{O} 2 p$-states and $\Delta E_{\mathrm{O}}-\Delta E_{\mathrm{OH}}$, we turn to DFT calculations of model FCC (111) transition metal surfaces with either $\mathrm{O}$ or $\mathrm{OH}$ adsorbed. In all cases, the metal atoms are fixed to their bulk positions and the $\mathrm{M}-\mathrm{O}$ bond distance is varied to simulate different $\mathrm{M}-\mathrm{O}$ coupling strengths. The in-plane positions of oxygen and hydrogen are fixed to be directly above a surface metal atom, but the $\mathrm{O}-\mathrm{H}$ bond length is allowed to change. The results are displayed in Fig. 3 as a two-dimensional grid of PDOS plots where the metal $d$-band and $\mathrm{M}-\mathrm{O}$ coupling strength are systematically varied along either axis.
Moving from left to right across a row corresponds to moving from left to right across the transition metal $d$-block, from higher lying $d$-bands to lower lying $d$-bands relative to the Fermi level. Moving from top to bottom corresponds to decreasing the $\mathrm{M}-\mathrm{O}$ bond distance and thus increasing the $\mathrm{M}-\mathrm{O}$ overlap and coupling strength.

In each element of the grid, two PDOS spectra are shown: in gray, the $d$-states of a surface metal atom before oxygen is adsorbed and in color, the $2 p$-states projected onto adsorbed oxygen. Also indicated in each grid element are $\Delta E_{\mathrm{O}}-\Delta E_{\mathrm{OH}}$ and $\bar{\varepsilon}_{2 p}$ as defined by Eqs. (1) and (2), respectively. Examining the $\mathrm{O} 2 p$-states in the top left grid element, $\mathrm{Ti}$, the presence of $\mathrm{M}-\mathrm{O}$ bonding states primarily below the Fermi level and $\mathrm{M}-\mathrm{O}$ antibonding states primarily above the Fermi level are apparent. The peak due to bonding states is much stronger compared to the peak due to antibonding states, suggesting the bonding states are quite $\mathrm{O} 2 p$-like in character while the antibonding states are quite Ti $3 d$ like in character. The $d$-band model contends that as we move across the periodic table to the right, the metal's $d$-band shifts down, and as a result, the bonding and antibonding states also shift down. [2] This behavior is observed clearly by examining the top right grid element, $\mathrm{Au}$, where the antibonding states are now mostly occupied, indicative of a weak $\mathrm{Au}-\mathrm{O}$ bond. Also note that in the case of $\mathrm{Au}$, the bonding states are very metal $d$-like while the antibonding states are very $\mathrm{O} 2 p$ like, in contrast to the picture on the more reactive Ti. In general, as the $d$-band shifts up (moving from right to left in the grid), bonding states become more oxygen-like and antibonding states become more metallike, which is also manifested as a decrease in $\bar{\varepsilon}_{2 p}$. This is exactly as one would reason from the simple two-level quantum coupling problem and is illustrated schematically in Fig. 4. We note that in this schematic, it is assumed that the position of the renormalized O $2 p$-states is constant with respect to the Fermi level, i.e. that the nature of the interaction between metal $s p$-band and $\mathrm{O} 2 p$-states is similar between metals and that differences in metal work functions is negligible as has been assumed previously. [2] Considering differences in work function would likely magnify the described effect as metals with higher work functions (Au for example) have lower lying $d$-bands relative to the atomic $\mathrm{O} 2 p$ states, resulting in higher-lying renormalized $\mathrm{O} 2 p$-states and thus more $\mathrm{O} 2 p$-state character in the $\mathrm{M}-\mathrm{O}$ antibonding states. Finally, because higher $d$-bands lead to stronger (more negative) $\mathrm{M}-\mathrm{O}$ bonds, we arrive at a correlation between the M-O bond strength $\left(\Delta E_{\mathrm{O}}-\Delta E_{\mathrm{OH}}\right)$ and $\bar{\varepsilon}_{2 p}$ with respect to variations in the metal $d$-band. This is our understanding for how the average $2 p$-state energy of an adsorbed oxygen atom is 


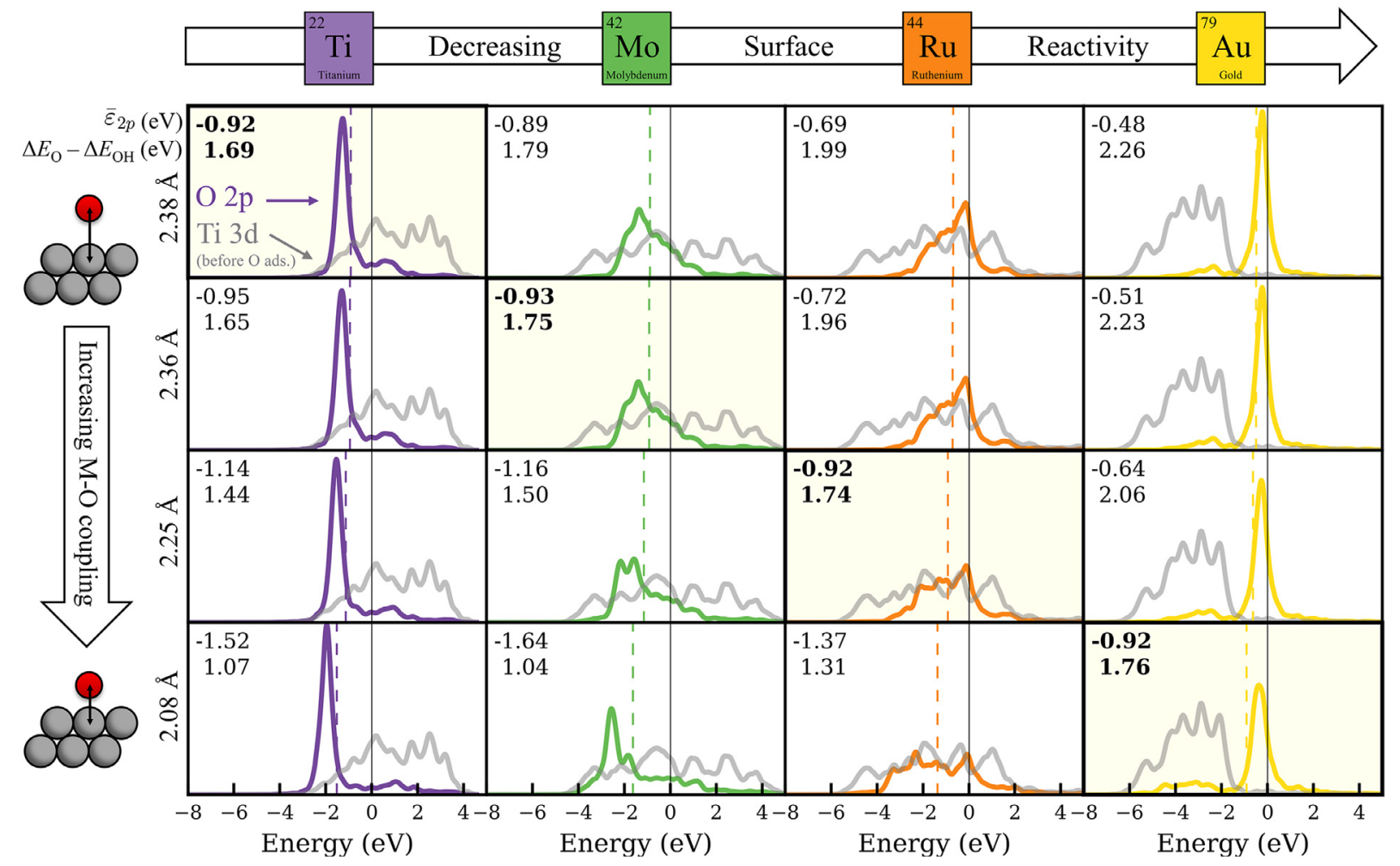

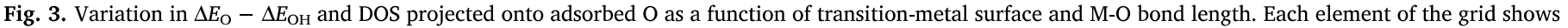

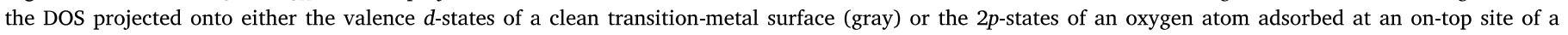

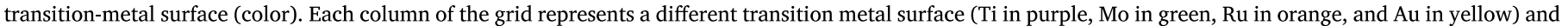

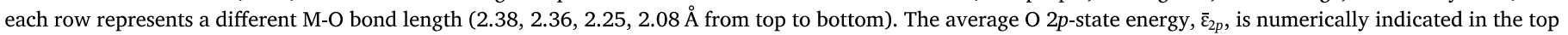

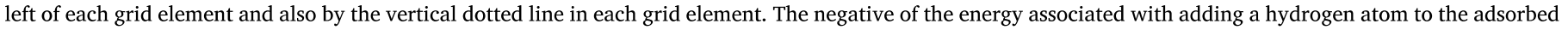

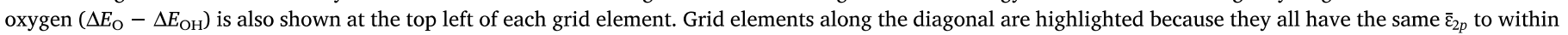

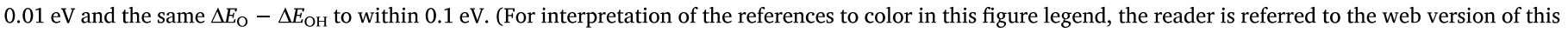
article.)

reflective of the electronic structure of the metal atom to which it is bound.

Next, we consider how changes in $\mathrm{M}-\mathrm{O}$ coupling strength affect the average $\mathrm{O} 2 p$-state energy and the $\mathrm{M}-\mathrm{O}$ bond strength. Moving down a column of the grid in Fig. 3 represents shortening the $\mathrm{M}-\mathrm{O}$ bond length, thereby systematically increasing the $\mathrm{M}-\mathrm{O}$ coupling strength.
Variations in the $\mathrm{O} 2 p$-states upon variations in bond length are subtle, but the dominant effect is an increase in oxygen-character for the bonding states for shorter bond lengths, resulting in a decrease in $\bar{\varepsilon}_{2 p}$. We hypothesize this effect is due to an increase in coupling between $O$ $2 p$-states and the metal's $s p$-band, resulting in lower lying renormalized $\mathrm{O} 2 p$-states (before coupling to the metal's $d$-band). In terms of the $\mathrm{M}-\mathrm{O}$
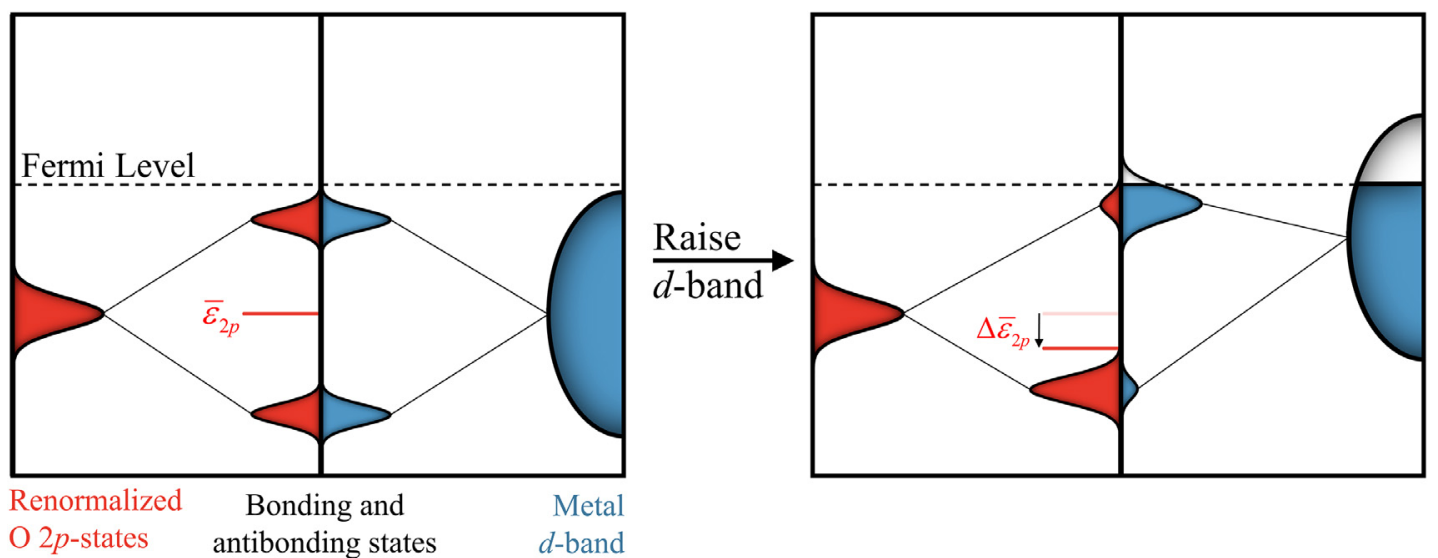

Fig. 4. Schematic illustrating how changes in the metal $d$-band are manifested in the $2 p$-states of adsorbed oxygen at a transition metal surface. Before coupling to the metal $d$-band, the $\mathrm{O} 2 p$-states couple to the metal $s p$-band, resulting in the renormalized $\mathrm{O} 2 p$-states shown at left of each energy diagram. These states couple with the metal $d$-band (shown at right) to form bonding and antibonding states (shown at center). These bonding and antibonding states are projected onto either the $\mathrm{O} 2 p$ states or metal $d$-states as indicated by the left-facing red peaks and right-facing blue peaks, respectively. The horizontal red line at center represents the average energy of the bonding and antibonding states projected onto the $\mathrm{O} 2 p$-states, $\bar{\varepsilon}_{2 p}$. (For interpretation of the references to color in this figure legend, the reader is referred to the web version of this article.) 


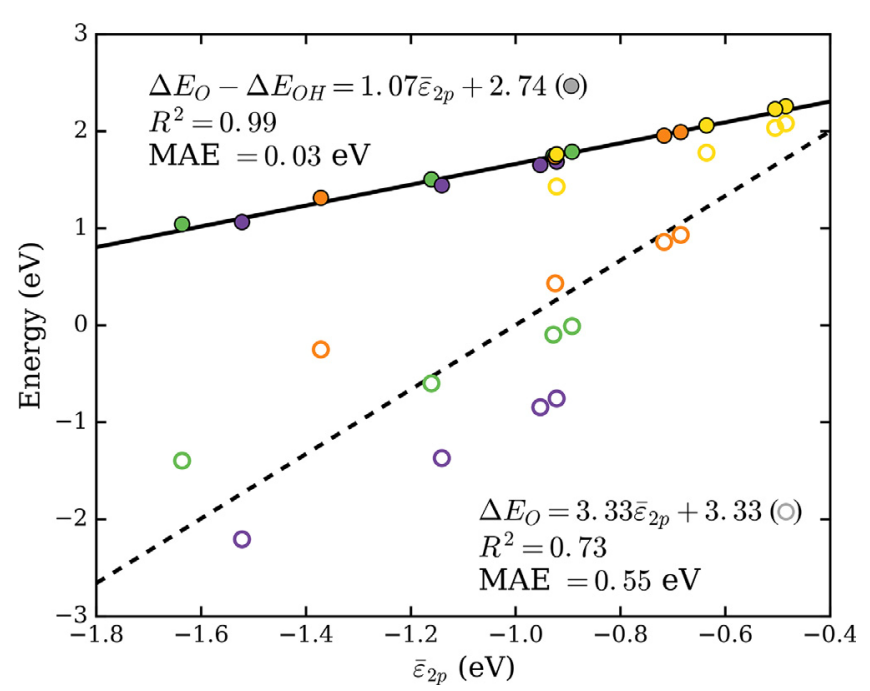

Fig. 5. Correlation between either $\Delta E_{\mathrm{O}}-\Delta E_{\mathrm{OH}}$ (filled) or $\Delta E_{\mathrm{O}}$ (unfilled) and $\bar{\varepsilon}_{2 p}$ for all grid elements in Fig. 3. An analogous figure comparing $\Delta E_{\mathrm{O}}-\Delta E_{\mathrm{OH}}$ and $\Delta E_{\mathrm{OH}}$ is shown in Fig. $\mathrm{S} 6$.

bond strength, decreasing the $\mathrm{M}-\mathrm{O}$ bond length (increasing coupling) results in a steady decrease of $\Delta E_{\mathrm{O}}-\Delta E_{\mathrm{OH}}$ (a stronger $\mathrm{M}-\mathrm{O}$ bond). Thus, we observe a positive correlation between $\bar{\varepsilon}_{2 p}$ and $\Delta E_{\mathrm{O}}-\Delta E_{\mathrm{OH}}$ with respect to variations in $\mathrm{M}-\mathrm{O}$ coupling strength.

The combined effect of the surface electronic structure and $\mathrm{M}-\mathrm{O}$ coupling strength on the average $\bar{\varepsilon}_{2 p}$ and $\Delta E_{\mathrm{O}}-\Delta E_{\mathrm{OH}}$ are illustrated in the diagonal of the grid in Fig. 3. Elements along the diagonal experience a trade-off between metal reactivity and $\mathrm{M}-\mathrm{O}$ coupling strength such that they all exhibit the same $\Delta E_{\mathrm{O}}-\Delta E_{\mathrm{OH}}$ to within $0.1 \mathrm{eV}$. Most importantly, through a convolution of the effects discussed above, they also have nearly identical average $\mathrm{O} 2 p$-state energies despite significant variations in the overall shape of the $\mathrm{O} 2 p$-state distributions. As shown in Fig. 5, all elements of the grid follow a nearly perfect correlation similar to those in Fig. 1 meaning that variations in $\bar{\varepsilon}_{2 p}$ translate directly to variations in $\Delta E_{\mathrm{O}}-\Delta E_{\mathrm{OH}}$ even for non-equilibrium geometries. We note that while the metal's $d$-band center also serves as a good descriptor of for variations in $\Delta E_{\mathrm{O}}-\Delta E_{\mathrm{OH}}$ with respect to variations in the metal's electronic structure, it is indifferent to changes in the M-O bond length as shown in Fig. S5.

This analysis illustrates the strength of the correlation between $\bar{\varepsilon}_{2 p}$ and $\Delta E_{\mathrm{O}}-\Delta E_{\mathrm{OH}}$ and provides some physical basis as to why it is robust to changes in oxygen binding site, which can be thought of as a specific mechanism of varying the $\mathrm{M}-\mathrm{O}$ coupling strength. Although we have primarily made a physical argument for transition metals, we hypothesize that this notion of $\bar{\varepsilon}_{2 p}$ being reflective of the surface electronic structure and $\mathrm{M}-\mathrm{O}$ coupling strength is responsible for its ability to describe the reactivity of oxygen at the surfaces of both metals and metal-oxides.

\subsection{Rationalizing the enhanced correlation of $\bar{\varepsilon}_{2 p}$ with $\Delta E_{O}-\Delta E_{O H}$ compared to $\Delta E_{O}$}

Fig. 5 shows that the correlation between $\bar{\varepsilon}_{2 p}$ and $\Delta E_{\mathrm{O}}-\Delta E_{\mathrm{OH}}$ is much stronger than the correlation between $\bar{\varepsilon}_{2 p}$ and $\Delta E_{\mathrm{O}}$ (also see Figs. S2 and S3). If variations in $\Delta E_{\mathrm{O}}-\Delta E_{\mathrm{OH}}$ are truly due to variations in the $\mathrm{M}-\mathrm{O}$ bond strength, then the question remains: why is $\bar{\varepsilon}_{2 p}$ a significantly better descriptor of $\Delta E_{\mathrm{O}}-\Delta E_{\mathrm{OH}}$ than $\Delta E_{\mathrm{O}}$ ? Fig. 6, which shows $\Delta E_{\mathrm{O}}, \Delta E_{\mathrm{OH}}$, and their difference as a function of $\mathrm{M}-\mathrm{O}$ bond length for $\mathrm{Ti}$ (111) over a wider range than in Fig. 5, provides some insight. While $\Delta E_{\mathrm{O}}$ and $\Delta E_{\mathrm{OH}}$ show typical chemisorption wells characteristic of the tradeoff between attractive hybridization and repulsive orthogonalization terms, $\Delta E_{\mathrm{O}}-\Delta E_{\mathrm{OH}}$ remains concave down across all the way down to a bond length of $1.2 \AA$ (as does $\bar{\varepsilon}_{2 p}$ ). This suggests that

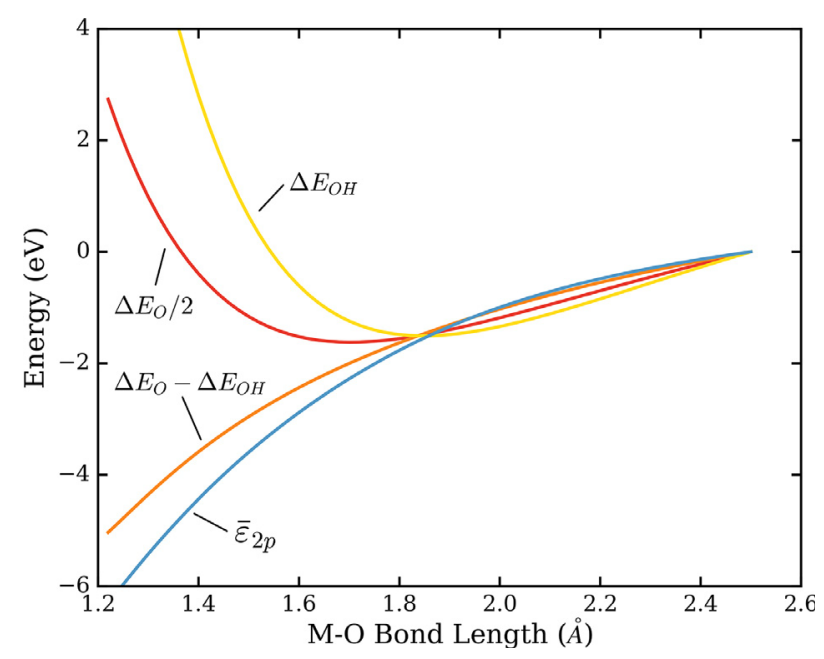

Fig. 6. $\Delta E_{\mathrm{O}}, \Delta E_{\mathrm{OH}}, \Delta E_{\mathrm{O}}-\Delta E_{\mathrm{OH}}$, and $\bar{\varepsilon}_{2 p}$ as a function of the $\mathrm{M}-\mathrm{O}$ bond length above a Ti (111) surface. As in Fig. 3, the in-plane coordinates of oxygen are fixed to that of a surface metal atom. All energies are relative to their values at a bond length of $2.5 \AA$.

variations in $\Delta E_{\mathrm{O}}-\Delta E_{\mathrm{OH}}$ are primarily due to variations in hybridization energy of the $\mathrm{M}-\mathrm{O}$ bond, or equivalently, that the orthogonalization energy of adsorbed $\mathrm{O}$ and adsorbed $\mathrm{OH}$ are similar. This is as expected since the orbital overlap between oxygen and metal is similar for either $\mathrm{O}$ or $\mathrm{OH}$ at a given $\mathrm{M}-\mathrm{O}$ bond length. Ultimately, because $\bar{\varepsilon}_{2 p}$ is consistently a better descriptor of $\Delta E_{\mathrm{O}}-\Delta E_{\mathrm{OH}}$ compared to $\Delta E_{\mathrm{O}}$ or $\Delta E_{\mathrm{OH}}$, we propose that the fundamental link identified in this work is between the average energy of the $O 2 p$-states and the hybridization energy of the $\mathrm{M}-\mathrm{O}$ bond (for which $\Delta E_{\mathrm{O}}-\Delta E_{\mathrm{OH}}$ is a good proxy):

$\delta \bar{\varepsilon}_{2 \mathrm{p}} \sim \delta E_{\mathrm{M}-\mathrm{O}}^{\mathrm{hyb}} \sim \delta\left(\Delta E_{\mathrm{O}}-\Delta E_{\mathrm{OH}}\right)$.

\section{Application: Screening of active sites}

Lastly, we will demonstrate some practical utility of $\bar{\varepsilon}_{2 p}$ as a descriptor, which allows one to predict the reactivity of any number of oxygen atoms present in a DFT calculation. This can be an effective screening tool for complicated oxide surfaces or nanoparticles consisting of many inequivalent oxygen sites. We take for example from our previous work a kinked $\mathrm{RuO}_{2}$ (121) surface, which we have hypothesized is representative of the active site for OER. [45] As shown in Fig. 7, there are many inequivalent oxygen sites present at this surface. Ignoring sites in the bottom two layers (which are fixed to their bulk positions), there are more than 30 unique surface and subsurface oxygen sites. The DOS projected onto each of these oxygen atoms was extracted from a single self-consistent DFT calculation and used to calculate the average $O 2 p$-state energy, $\bar{\varepsilon}_{2 p}$, which is indicated by the coloring of each oxygen in Fig. 7.

The slope of $\sim 1$ for the correlation in Fig. 1 suggests that the variations in $\bar{\varepsilon}_{2 p}$ should directly translate into variations in $\Delta E_{\mathrm{O}}-\Delta E_{\mathrm{OH}}$. In other words, based on the electronic structure of the oxygen covered surface $\mathrm{RuO}_{2}$ surface, we expect there to be a $\sim 1.5 \mathrm{eV}$ range in oxygen reactivity across its various oxygen sites. This prediction is confirmed at right in Fig. 7 , where $\bar{\varepsilon}_{2 p}$ and $\Delta E_{\mathrm{O}}-\Delta E_{\mathrm{OH}}$ are shown to track each other across the various sites.

As mentioned at the outset, $\Delta E_{\mathrm{O}}-\Delta E_{\mathrm{OH}}$ is the primary descriptor for determining the theoretical overpotential for the OER. Using the average $\mathrm{O} 2 p$-state energy, one can predict the OER activity for any number of oxygen atoms using the density of states from a single selfconsistent DFT calculation. Additionally, the $\bar{\varepsilon}_{2 p}$ of each site could be used in an iterative process to determine the most stable surface 


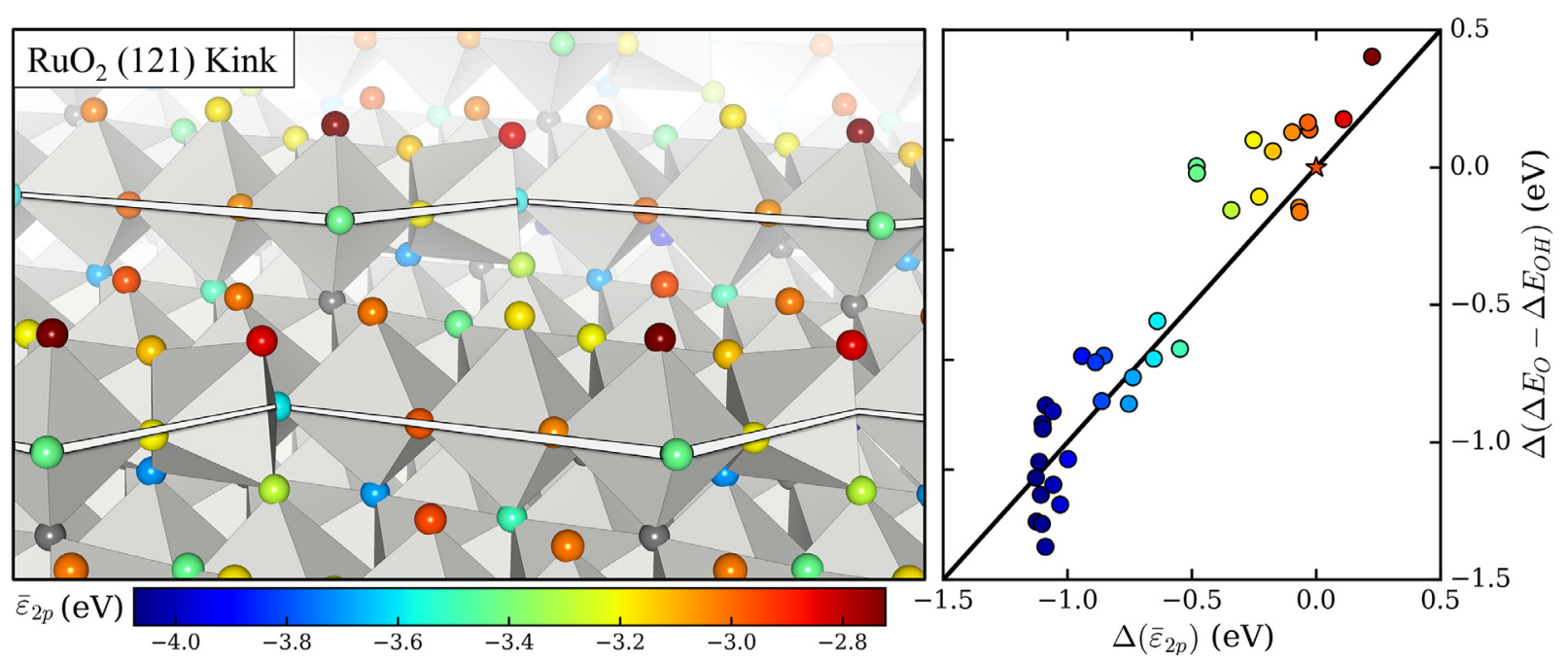

Fig. 7. (Left) Visualization of the average $2 p$-state energy, $\bar{\varepsilon}_{2 p}$, for each oxygen atom at a kinked $\mathrm{RuO}_{2}(121)$ surface. White bars are added to give perspective and help guide the eye along the kink/step edges). The average $2 p$-state energy for each oxygen (as extracted from a single self-consistent DFT calculation) is indicated by its color. Oxygen atoms colored gray were unable to support a hydrogen atom without transferring it to another site. (Right) A parity plot between variations in $\bar{\varepsilon}_{2 p}$ vs variations in $\Delta E_{\mathrm{O}}-\Delta E_{\mathrm{OH}}$. The oxygen site chosen as reference is indicated by a star and has an absolute $\bar{\varepsilon}_{2 p}$ of $-2.95 \mathrm{eV}$ and an absolute $\Delta E_{\mathrm{O}}-\Delta E_{\mathrm{OH}}$ of $1.26 \mathrm{eV}$. (For interpretation of the references to color in this figure legend, the reader is referred to the web version of this article.)

coverage under reaction conditions as explained in the Supporting Information. For large systems such as this one with many possible active sites, high accuracy predictions can yield significant savings of computational resources. For instance, structural relaxation of hydrogen adsorbed at each oxygen site in Fig. 7 required on average 2400 CPU hours, totaling $86 \mathrm{k}$ CPU hours for all 36 sites as compared to $<100 \mathrm{CPU}$ hours for a single self-consistent calculation of the oxygen covered surface. Active site screening on metal-oxide nanoparticles could be made feasible using the descriptor identified in this work, in a way analogous to generalized and orbitalwise coordination numbers for metal nanoparticles. $[11,13,15,16]$

\section{Conclusions}

In this work, we have demonstrated a robust correlation between the average $2 p$-state energy, $\bar{\varepsilon}_{2 p}$, and reactivity of surface oxygen atoms, $\Delta E_{\mathrm{O}}-\Delta E_{\mathrm{OH}}$, that holds across metal and metal-oxide surfaces and different oxygen binding sites. In other words, $\bar{\varepsilon}_{2 p}$ is a site-specific descriptor for surface oxygen reactivity possessing greater resolution than the bulk/subsurface $\mathrm{O} 2 p$-band center or charge-transfer energy, which have both been shown previously to correlate with experimental catalytic rates. $[27,29,30,32]$ We have utilized the $d$-band model to provide a physical basis for this correlation by considering how variations in $\mathrm{M}-\mathrm{O}$ coupling strength and the metal $d$-band center result in changes to both $\mathrm{M}-\mathrm{O}$ bond strength and $\mathrm{O} 2 p$-states at transition metal surfaces. Based on the fact that $\bar{\varepsilon}_{2 p}$ is a significantly better descriptor of $\Delta E_{\mathrm{O}}-\Delta E_{\mathrm{OH}}$ than $\Delta E_{\mathrm{O}}$ and that the former remains concave down as a function of $\mathrm{M}-\mathrm{O}$ bond length, we hypothesize that a fundamental link exists between the average $\mathrm{O} 2 p$-state energy and $\mathrm{M}-\mathrm{O}$ hybridization energy, for which $\Delta E_{\mathrm{O}}-\Delta E_{\mathrm{OH}}$ is a good proxy. Finally, we have demonstrated some practical utility of $\bar{\varepsilon}_{2 p}$ as a descriptor by predicting the reactivity of every oxygen site on an extended, heterogeneous surface structure, kinked $\mathrm{RuO}_{2}$ (121), from a single self-consistent DFT calculation. Such a strategy has the potential to significantly accelerate the identification of active sites at complex metal-oxide surfaces or nanoparticles where individually measuring the reactivity of each oxygen site comes with significant computational cost.

\section{Acknowledgments}

This work was supported by a grant of the U.S. Department of
Energy, Office of Science, Office of Basic Energy Sciences, via Grant DESC0008685 to the SUNCAT Center for Interface Science and Catalysis and the Villum Fonden. The authors acknowledge the use of the computer time allocation for "transition metal-oxide and metal surfaces: applications and reactivity trends in catalysis," at the National Energy Research Scientific Computing Center, a DOE Office of Science User Facility supported by the Office of Science of the U.S. Department of Energy under Contract No. DE-AC02-05CH11231. Support for C.F.D. was provided by the National Science Foundation Graduate Research Fellowship (Grant DGE-114747). The authors acknowledge helpful discussion with Allegra Latimer. Structures in Figs. 1 and 7 were rendered with the Atomic Simulation Environment and VESTA, respectively. $[39,46]$ All quantitative figures were created with the Matplotlib python package [47].

\section{References}

[1] B. Hammer, J.K. Norskov, Why gold is the noblest of all the metals, Nature 376 (1995) 238-240, https://doi.org/10.1038/376238a0.

[2] B. Hammer, J.K. Nørskov, Electronic factors determining the reactivity of metal surfaces, Surf. Sci. 343 (1995) 211-220, https://doi.org/10.1016/0039-6028(96) 80007-0.

[3] B. Hammer, J.K. Norskov, Theoretical surface science and catalysis - calculation and concepts, Adv. Catal. 45 (2000) 71-129 http://dx.doi.org/10.1016/S03600564(02)45013-4.

[4] J. Greeley, M. Mavrikakis, Alloy catalysts designed from first principles, Nat. Mater. 3 (2004) 810-815, https://doi.org/10.1038/nmat1223.

[5] J.R. Kitchin, J.K. Nørskov, M.A. Barteau, J.G. Chen, Modification of the surface electronic and chemical properties of $\mathrm{Pt}(111)$ by subsurface $3 \mathrm{~d}$ transition metals, J. Chem. Phys. 120 (2004) 10240-10246, https://doi.org/10.1063/1.1737365.

[6] M. Mavrikakis, B. Hammer, J.K. Nørskov, Effect of strain on the reactivity of metal surfaces, Phys. Rev. Lett. 81 (1998) 2819-2822, https://doi.org/10.1103/ PhysRevLett.81.2819.

[7] T. Jiang, D.J. Mowbray, S. Dobrin, H. Falsig, B. Hvolbøk, T. Bligaard, J.K. Nørskov, Trends in CO oxidation rates for metal nanoparticles and close-packed, stepped, and kinked surfaces, J. Phys. Chem. C 113 (2009) 10548-10553, https://doi.org/10. 1021/jp811185g.

[8] F. Abild-Pedersen, J. Greeley, F. Studt, J. Rossmeisl, T.R. Munter, P.G. Moses, E. Skúlason, T. Bligaard, J.K. Nørskov, Scaling properties of adsorption energies for hydrogen-containing molecules on transition-metal surfaces, Phys. Rev. Lett. 99 (2007) 016105, , https://doi.org/10.1103/PhysRevLett.99.016105.

[9] J.K. Nørskov, T. Bligaard, J. Rossmeisl, C.H. Christensen, Towards the 
computational design of solid catalysts, Nat. Chem. 1 (2009) 37-46, https://doi. org $/ 10.1038 /$ nchem. 121 .

[10] T. Bligaard, J.K. Nørskov, S. Dahl, J. Matthiesen, C.H. Christensen, J. Sehested, The Brønsted-Evans-Polanyi relation and the volcano curve in heterogeneous catalysis, J. Catal. 224 (2004) 206-217, https://doi.org/10.1016/j.jcat.2004.02.034.

[11] F. Calle-Vallejo, J.I. Martínez, J.M. García-Lastra, P. Sautet, D. Loffreda, Fast prediction of adsorption properties for platinum nanocatalysts with generalized coordination numbers, Angew. Chemie Int. Ed. 53 (2014) 8316-8319, https://doi org/10.1002/anie.201402958.

[12] F. Calle-Vallejo, D. Loffreda, M.T.M. Koper, P. Sautet, Introducing structural sensitivity into adsorption-energy scaling relations by means of coordination numbers, Nat. Chem. 7 (2015) 403-410, https://doi.org/10.1038/nchem.2226.

[13] F. Calle-Vallejo, J. Tymoczko, V. Colic, Q.H. Vu, M.D. Pohl, K. Morgenstern, D. Loffreda, P. Sautet, W. Schuhmann, A.S. Bandarenka, Finding optimal surface sites on heterogeneous catalysts by counting nearest neighbors, Science 350 (2015) 185-189, https://doi.org/10.1126/science.aab3501.

[14] L.T. Roling, F. Abild-Pedersen, Structure-sensitive scaling relations: adsorption energies from surface site stability, ChemCatChem. 10 (2018) 1643-1650, https:// doi.org/10.1002/cctc. 201701841 .

[15] X. Ma, H. Xin, Orbitalwise coordination number for predicting adsorption properties of metal nanocatalysts, Phys. Rev. Lett. 118 (2017) 1-5, https://doi.org/10. 1103/PhysRevLett.118.036101.

[16] S. Wang, N. Omidvar, E. Marx, H. Xin, Overcoming site heterogeneity in search of metal nanocatalysts, ACS Comb. Sci. 20 (2018) 567-572, https://doi.org/10.1021/ acscombsci.8b00070.

[17] V. Fung, F.F. Tao, D Jiang, General structure-reactivity relationship for oxygen on transition-metal oxides, J. Phys. Chem. Lett. (2017) 2206-2211, https://doi.org/10 1021/acs.jpclett.7b00861.

[18] H. Xu, D. Cheng, D. Cao, X.C. Zeng, A universal principle for a rational design of single-atom electrocatalysts, Nat. Catal. 1 (2018) 339-348, https://doi.org/10. 1038/s41929-018-0063-z.

[19] D. Wu, C. Dong, H. Zhan, X. Du, Bond-energy-integrated descriptor for oxygen electrocatalysis of transition metal oxides, J. Phys. Chem. Lett. 9 (2018) 3387-3391, https://doi.org/10.1021/acs.jpclett.8b01493.

[20] J. Suntivich, K.J. May, H.A. Gasteiger, J.B. Goodenough, Y. Shao-Horn, A perovskite oxide optimized for oxygen evolution catalysis from molecular orbital principles, Science 334 (2011) 1383-1385, https://doi.org/10.1126/science.1212858.

[21] F. Calle-Vallejo, N.G. Inoglu, H.Y. Su, J.I. Martínez, I.C. Man, M.T.M. Koper, J.R. Kitchin, J. Rossmeisl, Number of outer electrons as descriptor for adsorption processes on transition metals and their oxides, Chem. Sci. 4 (2013) 1245-1249, https://doi.org/10.1039/c2sc21601a.

[22] S.A. Akhade, J.R. Kitchin, Effects of strain, d-band filling, and oxidation state on the surface electronic structure and reactivity of $3 \mathrm{~d}$ perovskite surfaces, J. Chem. Phys. 137 (2012) 084703, , https://doi.org/10.1063/1.4746117.

[23] M. García-Mota, A. Vojvodic, F. Abild-Pedersen, J.K. Nørskov, Electronic origin of the surface reactivity of transition-metal-doped TiO 2 (110), J. Phys. Chem. C 117 (2013) 460-465, https://doi.org/10.1021/jp310667r.

[24] Z. Xu, J.R. Kitchin, Relating the electronic structure and reactivity of the 3d transition metal monoxide surfaces, Catal. Commun. 52 (2014) 60-64, https://doi.org/ 10.1016/j.catcom.2013.10.028.

[25] Z. Xu, J.R. Kitchin, Relationships between the surface electronic and chemical properties of doped $4 \mathrm{~d}$ and $5 \mathrm{~d}$ late transition metal dioxides, J. Chem. Phys. 142 (2015), https://doi.org/10.1063/1.4914093.

[26] A. Vojvodic, J. Norskov, Optimizing perovskites for the water splitting reaction, Science 1334 (2011) 1355, https://doi.org/10.1126/science.1215081.

[27] J.H. Montoya, A.D. Doyle, J.K. Nørskov, A. Vojvodic, Trends in adsorption of electrocatalytic water splitting intermediates on cubic ABO 3 oxides, Phys. Chem. Chem. Phys. 20 (2018) 3813-3818, https://doi.org/10.1039/C7CP06539F.

[28] J. Suntivich, K.J. May, H.A. Gasteiger, J.B. Goodenough, Y. Shao-Horn, A perovskite oxide optimized for oxygen evolution catalysis from molecular orbital principles, Science 334 (2011) 1383-1385, https://doi.org/10.1126/science.1212858.

[29] Y.L. Lee, J. Kleis, J. Rossmeisl, Y. Shao-Horn, D. Morgan, Prediction of solid oxide fuel cell cathode activity with first-principles descriptors, Energy Environ. Sci. 4 (2011) 3966, https://doi.org/10.1039/c1ee02032c.
[30] A. Grimaud, K.J. May, C.E. Carlton, Y.-L. Lee, M. Risch, W.T. Hong, J. Zhou, Y. Shao-Horn, Double perovskites as a family of highly active catalysts for oxygen evolution in alkaline solution, Nat. Commun. 4 (2013) 2439, https://doi.org/10. 1038/ncomms3439.

[31] W.T. Hong, R.E. Welsch, Y. Shao-Horn, Descriptors of oxygen-evolution activity for oxides: a statistical evaluation, J. Phys. Chem. C (2015), https://doi.org/10.1021/ acs.jpcc.5b10071 acs.jpcc.5b10071.

[32] W. Hong, K.A. Stoerzinger, Y.-L. Lee, L. Giordano, A.J.L. Grimaud, A.M. Johnson, J. Hwang, E. Crumlin, W. Yang, Y. Shao-Horn, Charge-transfer-energy-dependent oxygen evolution reaction mechanisms for perovskite oxides, Energy Environ. Sci. 10 (2017) 2190-2200, https://doi.org/10.1039/C7EE02052J.

[33] J. Graetz, A. Hightower, C.C. Ahn, R. Yazami, P. Rez, B Fultz, Electronic structure of chemically-delithiated LiCoO 2 studied by electron energy-loss spectrometry, J. Phys. Chem. B 106 (2002) 1286-1289, https://doi.org/10.1021/jp0133283.

[34] D. Ensling, G. Cherkashinin, S. Schmid, S. Bhuvaneswari, A. Thissen, W. Jaegermann, Nonrigid band behavior of the electronic structure of $\mathrm{LiCoO} 2$ thin film during electrochemical Li deintercalation, Chem. Mater. 26 (2014) 3948-3956, https://doi.org/10.1021/cm501480b.

[35] I.C. Man, H.-Y. Su, F. Calle-Vallejo, H.a. Hansen, J.I. Martínez, N.G. Inoglu, J. Kitchin, T.F. Jaramillo, J.K. Nørskov, J. Rossmeisl, Universality in oxygen evolution electrocatalysis on oxide surfaces, ChemCatChem 3 (2011) 1159-1165, https://doi.org/10.1002/cctc. 201000397.

[36] A.A. Latimer, A.R. Kulkarni, H. Aljama, J.H. Montoya, J.S. Yoo, C. Tsai, F. Abild pedersen, F. Studt, J.K. Nørskov, Understanding trends in $\mathrm{C}-\mathrm{H}$ bond activation in heterogeneous catalysis, Nat. Mater. 16 (2017) 225-230, https://doi.org/10.1038/ NMAT4760.

[37] Z.W. Seh, J. Kibsgaard, C.F. Dickens, I. Chorkendorff, J.K. Nørskov, T.F. Jaramillo, Combining theory and experiment in electrocatalysis: Insights into materials design, Science 355 (2017) eaad4998, https://doi.org/10.1126/science.aad4998.

[38] P. Giannozzi, S. Baroni, N. Bonini, M. Calandra, R. Car, C. Cavazzoni, D. Ceresoli, G.L. Chiarotti, M. Cococcioni, I. Dabo, A. Dal Corso, S. de Gironcoli, S. Fabris, G. Fratesi, R. Gebauer, U. Gerstmann, C. Gougoussis, A. Kokalj, M. Lazzeri, L. Martin-Samos, N. Marzari, F. Mauri, R. Mazzarello, S. Paolini, A. Pasquarello, L. Paulatto, C. Sbraccia, S. Scandolo, G. Sclauzero, A.P. Seitsonen, A. Smogunov, P. Umari, R.M. Wentzcovitch, QUANTUM ESPRESSO: a modular and open-source software project for quantum simulations of materials, J. Phys. Condens. Matter 21 (2009) 395502, , https://doi.org/10.1088/0953-8984/21/39/395502.

[39] S.R. Bahn, K.W. Jacobsen, An object-oriented scripting interface to a legacy electronic structure code, Comput. Sci. Eng 4 (2002) 56-66, https://doi.org/10.1109/ 5992.998641.

[40] B. Hammer, L. Hansen, J. Nørskov, Improved adsorption energetics within density functional theory using revised Perdew-Burke-Ernzerhof functionals, Phys. Rev. B 59 (1999) 7413-7421, https://doi.org/10.1103/PhysRevB.59.7413.

[41] J. Wellendorff, K.T. Lundgaard, A. Møgelhøj, V. Petzold, D.D. Landis, J.K. Nørskov, T. Bligaard, K.W. Jacobsen, Density functionals for surface science: exchange-correlation model development with Bayesian error estimation, Phys. Rev. B 85 (2012) 235149, , https://doi.org/10.1103/PhysRevB.85.235149.

[42] Catalysis-Hub, https://www.catalysis-hub.org/publications/ DickensElectronic2018, (2018)

[43] J. Rossmeisl, A. Logadottir, J.K. Nørskov, Electrolysis of water on (oxidized) metal surfaces, Chem. Phys. 319 (2005) 178-184, https://doi.org/10.1016/j.chemphys. 2005.05.038.

[44] J. Rossmeisl, Z.-W. Qu, H. Zhu, G.-J. Kroes, J.K. Nørskov, Electrolysis of water on oxide surfaces, J. Electroanal. Chem. 607 (2007) 83-89, https://doi.org/10.1016/j. jelechem.2006.11.008.

[45] C.F. Dickens, J.K. Nørskov, A theoretical investigation into the role of surface defects for oxygen evolution on RuO 2, J. Phys. Chem. C 121 (2017) 18516-18524, https://doi.org/10.1021/acs.jpcc.7b03481.

[46] K. Momma, F. Izumi, VESTA 3 for three-dimensional visualization of crystal, volumetric and morphology data, J. Appl. Crystallogr. 44 (2011) 1272-1276, https:// doi.org/10.1107/S0021889811038970.

[47] J.D. Hunter, Matplotlib: a 2D graphics environment, Comput. Sci. Eng. 9 (2007) 90-95, https://doi.org/10.1109/MCSE.2007.55. 